\section{CASE OF POISONING BY CARBOLIC ACID.*}

BY GEORGE J. HEARDER, M.D.,

Medical Superintendent of the Lunatic Asylum, Carmarthen.

ABOUT ten o'clock on the morning of November 27th, I was called to see a man aged 36 , who had swallowed carbolic acid with suicidal intent. He said that he had "swallowed poison". About five minutes afterwards, when I first saw him, he was evidently in mortal agony. His countenance was livid, his eyeballs protruded, and he uttered a continuous subdued cry, of wild and fear-inspiring tone, which was broken only by short gasping attempts at respiration, and ineffectual endeavours to vomit. This condition was followed almost immediately by a state of profound insensibility. Olive oil was poured into his mouth while the stomach-pump was being prepared. Warm water was then injected into the stomach, and withdrawn strongly impregnated with carbolic acid. A second injection of olive oil was administered, and this likewise, when extracted, brought with it a portion of the poison. The patient died, apparently asphyxiated, about thirty minutes after taking the acid.

From the first the pulse was very rapid, and so feeble as to be scarcely perceptible ; the breathing was performed only by short and infrequent gasps, and the surface of the body was of a deep livid hue; the pupils were unaffected. Great difficulty was experienced in passing the gum elastic tube into the stomach. It was necessary to keep the forefinger of the left hand at the back of the pharynx, to prevent it curling up, while persistent and somewhat forcible pressure was employed to overcome the resistance made by the firmly contracted state of the œsophageal muscle. It falls to the lot of asylum medical officers to use the stomach-pump much more frequently than their brethren in general practice, and I have employed the stomach-pump on many hundred occasions as an aid in forced alimentation. In none of these was there any difficulty in passing the tube into the stomach after its point had got beyond the muscles of the pharynx. In the case under consideration, however, there was very considerable resistance offered by the cesophagus throughout its entire length ; and great care and perseverance were required to perform what is usually a very simple and easy operation.

Sectio Cadaveris Tiventy-six Hours after Death. - The superficial veins, especially those of the upper extremities, were distended with blood. The lips and chin were slightly excoriated. The dura mater was much congested with dark venous blood. The arachnoid at the vertex was thickened by gelatinous deposit. The brain-substance was apparently healthy. The lungs, filling well their cavities, were slightly emphysematcus in front, and passively congested posteriorly; there were old adhesions at both apices. The pericardial sac contained about an ounce of serum. The heart was healthy ; all its cavities were full of blood, which was very dark, perfectly fluid, and without a trace of coagula. Its microscopic examination revealed nothing abnormal. The mucous membrane of the larynx, trachea, and its subdivisions, was highly congested, granular, and softened; the tubes contained small quantities of olive oil and carbolic acid. The tongue appeared shrunken, pale, and indurated. The papillæ were markedly prominent; the len. ticular papillæ particularly so. The mucous membrane of the œesophagus was peculiarly white and glistening, softened, and peeling off as if scalded. The inner surface of the stomach was extensively softened and eroded, its mucous and muscular coats being reduced to a state of pulp. The parts surrounding the orifices had sustained most injury, and these portions were of a dull deep slate colour. The small intestine was similarly affected, in patches of about the size of a crownpiece, to within a few inches of the cæcum. The liver, spleen, and kidneys were healthy.

That the fatal issue was hastened by the action of the acid on the air-passages there can be no doubt ; and we have, in the same cause, also an explanation of the asphyxial symptoms and the rapidly supervening state of coma. Two cases of poisoning by carbolic acid have recently been reported in the JoURNAL of the Association. The first, that of a man who drank by mistake a glass of a mixture containing between two and three drachms of carbolic acid. He immediately fell insensible and was convulsed. Eighteen minutes afterwards, when seen by Dr. Mosler, the extremities were cold, the pulse scarcely perceptible; the heart's action was irregular, the breathing stertorous; consciousness was lost, and he was in a state of intense trismus. After evacuating the stomach, finding consciousness did not return, Dr. Mosler, supposing that some of the carbolic acid had entered the blood and was acting on the brain, bled the man to the amount of a pint.

* Read before the South Wales and Monmouthshire Branch.
Consciousness then returned. The patient was dismissed cured on the eleventh day.

The second case is reported as one of "poisoning from the fumes of carbolic acid". When first seen by the surgeon, the patient was in violent convulsions with trismus, and blood passing from the mouth in consequence of the teeth having wounded the tongue. He was placed in a warm bath, and in forty minutes the convulsions ceased. Convalescence was uninterrupted.

The patient under my care had taken probably an ounce of the undiluted acid. He became insensible about five minutes afterwards. There was no trismus, no convulsions, no stertor, but complete paralysis of all the voluntary muscles. I did not employ venesection, and the post mortem examination showed it would have been useless. And, further, I cannot comprehend in what manner venesection can counteract the effects of poisoned blood acting on the brain.

\section{A CASE OF FEMORAL ANEURISM CLOSELY SIMU- LATING MALIGNANT DISEASE.}

By GEORGE ALEXANDER GLOAG, L.K.Q.C.P., Bristol.

EDWARD P., aged 37, of a cachectic appearance, by occupation a pedlar, came under my care on November 5 th, 1872 , for the treatment of a tumour which occupied the anterior and inner region of the upper half of the right thigh. It was bounded above by Poupart's ligament, and had a circumference of $27 \frac{1}{4}$ inches at its centre, the circumference of the sound limb at the same part being 16 inches. The tumour had a tense elastic feel and a shiny appearance, the superficial veins were enlarged and prominent, and the disease appeared to have involved all the structures of the limb. No briit or pulsation could at any time be discovered in it. It gradually increased in size, and on December 20 th had attained a circumference of 30 inches. The patient suffered intense pain, which was of a paroxysmal character, and required. large doses of morphia or chloral for its relief. During severe pain I found that the tumour became harder, and that it increased in circumference to the extent of half an inch, and again subsided as the pain diminished to its former dimension. The limb was œedernatous below the tumour, the result of venous obstruction. Although there were no glandular enlargements nor symptoms of secondary deposit, the cachectic appearance of the patient, the intense pain he suffered, and the rapid growth of the tumour, together with the total absence of pulsation or stethoscopic sound, induced me to believe the case to be one of medullary cancer, for which operative interference was unjustifiable. About six months previously to the time when the patient came under my notice, a tumour, about the size of a small egg, appeared on the upper and inner side of the thigh, accompanied with such severe pain. that the patient was unable to follow his occupation, and was obliged to remain in bed. It grew rapidly from week to week, and the pain increased in proportion. A month or so after the appearance of the tumour, he obtained admission into the Bristol General Hospital. He remained there six weeks, during which time the tumour increased considerably in size, and was then discharged as an incurable case, the tumour being considered of a malignant nature. The patient remained. at home about a month, at the termination of which time he was admitted a patient of the Bristol Royal Infirmary. His case being considered one for which nothing could be done, he was removed to his own home in about a fortnight. The patient had usually enjoyed good health, and his family history was good; but he had suffered from constitutional syphilis, and was discharged from the army in consequence of defective vision, the result of specific iritis. There were no. evidences of heart-disease.

The patient died on December 28th, and on the following day Dr. Norton, Mr. Dobson, and myself made an examination of the body. The knee was bent, the thigh everted, and free movement existed at the hip-joint. An incision was made from the anterior superior spine of the ilium to the symphysis pubis, and another from the centre of Poupart's ligament down the front of the thigh. The latter was afterwards prolonged across the inner aspect of the knee, so as to expose the upper part of the popliteal space. On making the longitudinal incision, the parts gaped widely, and a thin layer of muscular tissue was exposed. On dividing this the length of the thigh, a mass of clot presenting various shades of colour appeared. Some of it was partly laminated and of a firm consistence, and needed the assistance of the knife for its removal. Nearly fourteen pounds weight of clot was turned out of the cavity, which was bounded anteriorly and to its sides by the skin, a small amount of subcutaneous fat, and a thin layer of muscular tissue; above by Poupart's ligament; below by the quadriceps extensor tendon; and behind by the eroded femur, 